\title{
Erinnerungsfenster für Demenzkranke
}

Fabian Mertl ${ }^{1}$, Bastian Dewitz ${ }^{1}$, Uli Braas ${ }^{1}$, Arthur Glomb ${ }^{1}$, Alina Huldtgren $^{1,3}$, Anja Vormann ${ }^{2}$, Christian Geiger ${ }^{1}$

Fachbereich Medien ${ }^{1}$ und Fachbereich Design ${ }^{2}$, Hochschule Düsseldorf, Deutschland Human-Technology Interaction, Technische Universität Eindhoven, Niederlande ${ }^{3}$

\section{Zusammenfassung}

Gerade weniger mobile Menschen mit Demenz haben oft nicht mehr die Möglichkeit, Ihre Lieblingsorte zu besuchen. Da aber gerade diese Orte oftmals zur Erhaltung der Identität dienen, ist es wünschenswert, die Erinnerung an diese zu unterstützen. In einem Projekt zum Thema multimediale Unterstützung von Erinnerungen und Kommunikation bei Demenzkranken wurde ein Erinnerungsfenster entwickelt, das es Menschen mit Demenz erlaubt, personalisierte Fensterausblicke zu erleben. In diesem Artikel beschreiben wir die Entwicklung des Konzeptes, sowie mehrere Iterationen der Entwicklung, in denen in Interviews oder Feldtests Feedback von der Zielgruppe eingeholt wurde.

\section{$1 \quad$ Einleitung}

Eine gesellschaftspolitische Herausforderung der kommenden Jahre ist die Versorgung und Pflege der steigenden Zahl an Demenzkranken. Demenz bezeichnet die fortschreitende Entwicklung von Gedächtnisverlust, Desorientierung in Raum und Zeit und den Verlust von Persönlichkeit in höherem Stadium. Die Teilhabe und die Ausführung von bedeutungsvollen Aktivitäten und Interaktionen mit anderen Menschen und folglich die persönliche Lebensqualität wird stark einschränkt (Wood et al. 2009). Die Wahrnehmung von Demenz ist in den letzten Jahren durch politische Aufklärung sowie bereitgestellte Forschungsgelder, gerade auch im Bereich der Mensch-Technik Interaktion ${ }^{1}$, in Deutschland enorm gestiegen. Dadurch ist es möglich geworden, die Bedürfnisse und Nöte von Demenzkranken intensiver $\mathrm{zu}$ erforschen. In unserem Forschungsschwerpunkt Nutzerwelten ${ }^{2}$ untersuchen wir, wie durch gestalterisch-technische Lösungen die Erinnerung erhalten und die Kommunikation von Demenzkranken angeregt werden kann. Im Folgenden wird die Entwicklung eines personalisierbaren Fensterblicks mit Hilfe eines virtuellen Fensters dargestellt.

1 www.bmbf.de/foerderungen/23550.php

2 www.nutzerwelten.de (gefördert im Program ,FH-Struktur' des Landes NRW) 


\section{Verwandte Arbeiten}

Demenz verläuft sehr individuell, obgleich sich grobe Stufen (leichte, mittel-schwere und schwere Demenz) erkennen lassen. Dennoch sollten Hilfsmittel individualisierbar bereitgestellt werden. Aufgrund eines Mangels an medikamentösen Behandlungen, sind psychosoziale Interventionen von großer Bedeutung und die Forschung hat gezeigt, dass u.A. durch Reminiszenz und biografische Arbeit, das psychische Wohlbefinden gestärkt wird (Godley \& Gatz 2000). Oftmals ist das episodische Langzeitgedächtnis von der Krankheit erst im letzten Stadium beeinträchtigt. Außerdem hilft Reminiszenz im Aufbau und Erhalt von Beziehung (Woods et al. 1992). Unsere Arbeit beruht auf Ergebnissen der Arbeit über den Einsatz von Technik in der Reminiszenztherapie (Lazar 2014). Die Entwicklung eines virtuellen Fensters beruht außerdem auf früheren Erkenntnissen (Friedman et. al 2008, Kahn et al. 2008), die in ihren Studien eine positive psychologische und biologische Wirkung auf Betrachter eines virtuellen Fensters im Arbeitsumfeld feststellen konnten.

\section{Entwicklung des Erinnerungsfenster}

Um den individuellen Bedürfnissen von Demenzkranken Güte tragen zu können, stand der Nutzer nach dem Prinzip des User-Centred Design im Mittelpunkt der Entwicklung. Erster Schritt war die Erfassung der Bedürfnisse und Fähigkeiten der Nutzer sowie deren Umfeld. In Kooperation mit dem Demenzservicezentrum war es möglich, Interviews mit Bewohnern und Personal in einer Pflegeeinrichtung zu führen, diese in die Entwicklung einzubeziehen und Zwischenergebnisse zu evaluieren.

\subsection{Konzept}

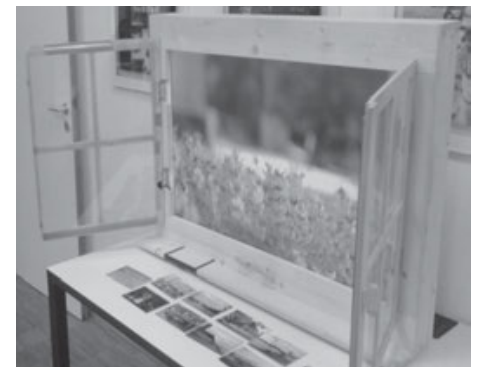

Abbildung 1: Erinnerungsfenster mit Postkarten zur Interaktion

Das Erinnerungsfenster soll Demenzkranken (1./2. Stufe) in Pflegeeinrichtungen, durch ein virtuelles Fenster bekannte Ausblicke in Bild und Ton erlauben. Zusätzlich soll es Angehörigen und Pflegern als Mediationsebene zur Kommunikation dienen.

Inhalt: Ziel ist es, personalisierte Aussichten anbieten zu können, die an bekannte Orte erinnern und somit zur Kommunikation mit anderen anregen. Die Ausblicke sollen Aufnahmen sein, die vom Personal aus Archiven oder von Verwandten in das System eingepflegt werden können. Vorrausetzung hierbei ist die Biografiearbeit von Pflegern sowie hochwertiges Filmmaterial in Full-HD Auflösung, das ruckelfrei mit einem stationären Blickwinkel über mehrere Minuten aufgenommen wurde, um einem Fensterblick zu ähneln. Das Fenster ist als Metapher zu sehen, durch die der Eindruck der Ausblicke in seiner Wirkung auf den Patienten verstärkt wird. Daher wurde ein Holzrahmen mit Fensterflügeln eigens für das Projekt angepasst (siehe Abb. 1). Um die Metapher zu unterstützen, werden die aufgenommenen Geräusche proportional lauter, wenn die Fensterflügel geöffnet werden. 
Eine realistische Bildverschiebung bei verschiedenen Betrachtungswinkeln könnte in Zukunft zur Verstärkung der Metapher auch noch berücksichtigt werden.

Wird kein personalisierter Inhalt abgerufen, wird auf dem Fenster allgemeiner Inhalt wiedergegeben, der sich dem Tag - Nacht Rhythmus anpasst. So bietet das Fenster den Patienten eine subtile Stütze, um sich diesem anzupassen und ähnelt einem echten Fenster. Inhalte wurden in mehreren Interviews mit zwei Bewohnerinnen konkretisiert. Durch das Verwenden beispielhafter Bilder in abstrakter, malerischer und fotografischer Form konnte auch ein Stil gefunden werden, der Menschen mit Demenz entgegenkommt. Vor Allem die fotografischen Bilder fanden Interesse und regten zur Kommunikation an. Abstrakte Bilder verunsicherten in Ihrer Bedeutung. Zu bevorzugten Themen gehörten Bilder der Gegend und Stadt sowie Naturaufnahmen mit Tieren. Bilder mit vielen Handlungen waren problematisch.

Interaktion: Der Wechsel des Inhalts wird durch das Auflegen bedruckter (RFID-gechipter) Postkarten auf dem Fensterbrett herbeigeführt. Die Postkarten zeigen allgemeine Szenen oder Portraits für personalisierten Inhalt. Ein Interface zum einfachen Hinzufügen neuer Videoinhalte und Verknüpfen mit RFID-Chips steht für die Pfleger zur Verfügung.

Technik: Das Display entspricht der Größe eines mittelgroßen Fensters. Die Technik sollte sich unauffällig hinter dem Fenster anbringen lassen. Verwendet wurde ein Minicomputer, RasperryPi Model B, zur Medienausgabe, der sich optimal verstecken ließ.

\section{Nutzerstudien}

Bereits während der Entwicklung wurden kleinere Studien durchgeführt, um z.B. die Interaktion und den Effekt des Fensters zu testen. Ein funktionsfähiger Prototyp wurde sowohl in einer Fokusgruppe mit Pflegern diskutiert als auch in Feldstudien getestet. Daten wurden durch Audioaufnahmen, Beobachtungsprotokolle und Feldnotizen erhoben.

Fokusgruppe. Im Gespräch mit vier Pflegern wurden Inhalte sowie Einsatzmöglichkeiten diskutiert. So ist es wichtig, dass das Bildmaterial mit besonderer Nachsicht in Schnitttechnik und der Auswahl des Handlungsgeschehens bearbeitet und ausgewählt wird. Auf schnelle Schnitte und kurze Szenen reagieren die Demenzkranken sensibel je fortgeschrittener ihr Stadium ist. Auch die Anzahl der Handlungen im Motiv sollte reduziert sein, um eine kognitive Verarbeitung zu ermöglichen. Als Inhalt wurden von Pflegern Szenen aus beliebten Ausflugszielen, wie der Kirmes oder Museumsbesuchen vorgeschlagen, um das Gefühl der Teilhabe am Gesellschaftsleben zu unterstützen und Gesprächsstoff $\mathrm{zu}$ bieten. Thematisierst wurde ebenfalls der zirkadiane Rhythmus des Inhalts, der die Patienten positiv unterstützen könnte. Pfleger waren sehr an einer mobilen Lösung interessiert, um auch Bettlägerigen eine Teilnahmemöglichkeit zu bieten.

Nutzertests. Der finale Prototyp wurde in Feldstudien getestet. Zunächst wurden Gruppen von 3-5 Personen mit Demenz (1./2. Stufe) 3-4 Stunden beobachtet, nachdem das Fenster in einem Gemeinschaftsraum platziert wurde. Danach wurde das Fenster aktiv in eine Gruppe von 7 Patienten und Pflegern in eine Gesprächsrunde eingebunden. Außerdem wurde der 
Prototyp bei einem öffentlichen Event mit Bewohnern und Angehörigen erprobt. Die Inhalte zeigten zwar den Bewohnern bekannte Orte, waren aber noch nicht auf jede Einzelperson zugeschnitten. Der Inhalt begeisterte Patienten sowie Angehörige und regte zur Kommunikation an. Durch lange Szenen war es Nutzern möglich, den Inhalt in Ruhe zu kommentieren. Ebenfalls wurde festgestellt, dass der Ton das Bild in seiner Wirkung unterstützt, die Funktion, die Lautstärke durch die Fensterflügel zu erhöhen jedoch von keinem genutzt wurde, wobei nicht sicher ist, ob die Funktion allen Teilnehmern bewusst war. Es wurde auch diskutiert, ob die Fenstermetapher überhaupt einen Mehrwert hat, da die meisten Bewohner den Fernseher dahinter erkannten. Der Effekt des Rahmens auf Menschen mit schwerer Demenz soll in zukünftigen Studien noch getestet werden. Obwohl das Auflegen der Postkarten leicht von den Bewohnern erfasst wurde, ließ deren Immobilität es oft nicht zu. Eine mobile Lösung des Lesegerätes würde die Nutzer aktiver einbeziehen.

\section{$5 \quad$ Fazit}

Zur Unterstützung der Erinnerung und Kommunikationsanregung von Menschen mit Demenz wurde ein interaktives Erinnerungsfenster entwickelt sowie erste Ergebnisse aus Diskussionen mit Pflegern und Feldtests präsentiert. Diese Art der multimedialen Unterstützung Demenzkranker - auch mit anderen multimedialen Erinnerungsobjekten wird in Zukunft innerhalb unseres Forschungsschwerpunktes tiefergehend untersucht.

\section{Literaturverzeichnis}

Astell, A. J., Ellis, M. P., Bernardi, L., Alm, N., Dye, R., Gowans, G., \& Campbell, J. (2010). Using a touch screen computer to support relationships between people with dementia and caregivers. Interacting with Computers, 22(4), 267-275.

Friedman, B., Freier, N. G., Kahn, P. H., Lin, P., \& Sodeman, R. (2008). Office window of the future?-Field-based analyses of a new use of a large display.International Journal of HumanComputer Studies, 66(6), 452-465.

Kasl-Godley, J., \& Gatz, M. (2000). Psychosocial interventions for individuals with dementia: an integration of theory, therapy, and a clinical understanding of dementia. Clinical psychology review, 20(6), 755-782.

Kahn, P. H., Friedman, B., Gill, B., Hagman, J., Severson, R. L., Freier, N. G., ... \& Stolyar, A. (2008). A plasma display window?-The shifting baseline problem in a technologically mediated natural world. Journal of Environmental Psychology, 28(2), 192-199.

Lazar, A., Thompson, H., \& Demiris, G. (2014). A systematic review of the use of technology for reminiscence therapy. Health education \& behavior, 41(1 suppl), 51-61.

Wood, W., Womack, J., \& Hooper, B. (2009). Dying of boredom: An exploratory case study of time use, apparent affect, and routine activity situations on two Alzheimer's special care units. American Journal of Occupational Therapy,63(3), 337-350.

Woods, B., Portnoy, S., Head, D., \& Jones, G. (1992). Reminiscence and life review with persons with dementia: which way forward. Care giving in dementia, 137-161. 


\title{
Ethik in Technikforschung und Technikentwicklung: Erfahrungen
}

\author{
Stefan Brandenburg
}

Kognitionspsychologie und Kognitive Ergonomie, Technische Universität Berlin

\section{Zusammenfassung}

Die Berücksichtigung ethischer Aspekte in der Mensch-Technik Interaktion (MTI) spielt eine immer größere Rolle in Forschung und Entwicklung. Es gibt zahlreiche Ethikkodizes für Ingenieure und Anforderungen von Mittelgebern, aber keine empirischen Daten zu der Häufigkeit und Art von ethisch relevanten Problemen bei Forschungs- und Entwicklungsprojekten. Wie schwierig es für Forscher und Entwickler sein kann, sich um die Einhaltung ethischer Grundsätze zu kümmern, zeigt der vorliegende Beitrag punktuell auf. Er gibt einen beispielhaften Überblick über die ethischen Aspekte bei MTIbezogenen Forschungs- und Entwicklungsvorhaben, mit denen Forscher und Entwickler an der TU Berlin konfrontiert wurden. Die Ergebnisse zeigen auf, dass es wiederkehrende Probleme, wie die Weitergabe/Darstellung von Informationen gibt, denen möglicherweise mit dem Ansatz einer Gebrauchsethik begegnet werden kann. Weitere Erkenntnisse für die Praxis werden diskutiert.

\section{Einleitung}

Konfrontiert man Forscher und Praktiker aus dem Feld der Mensch-Technik Interaktion mit dem Anspruch, sie möchten auch ethische Aspekte bei ihren Projekten berücksichtigen, dann entgegnen die Gefragten häufig mit der Gegenfrage: Warum? Einigen erscheint der zusätzliche Aufwand, der bei der Beschäftigung mit dem Thema entsteht als unverhältnismäßig hoch, verglichen mit dessen Bedeutung im Rahmen der Erforschung und Entwicklung technischer Artefakte. Dabei gibt es durchaus Gründe, sich auch hier um ethische Aspekte der Arbeit zu kümmern. So bringen beispielsweise die Entwicklungen des hochautomatisierten und autonomen Fahrens große, noch ungelöste ethische Probleme bezüglich der Führungsund Verantwortlichkeitsaufteilung von Fahrer und Fahrzeug mit sich. Leistungsstarke Algorithmen schaffen es bereits heute, die Identität von Kunden aus sogenannten Metadaten von Kreditkarten (z. B. Ort und Zeit der Nutzung und Preis der Ware) mit nur vier zufälligen Datenpunkten zu rekonstruieren (de Montjoye et al. 2015) und einfachste, alltäglich Sensorik, wie beispielsweise Bewegungsmelder, reicht aus, um personalisierte Bewegungsprofile von vorbeilaufenden Menschen zu erstellen (Mundt et al. 2012). Da bei der Einführung neuer 
Technologien nicht unbedingt deutlich werden muss, welche ethischen Probleme sich möglicherweise durch deren Nutzung ergeben, sollten derartige Aspekte bereits bei der Entwicklung mitgedacht werden (vgl. Ropohl 1996). Darüber hinaus argumentiert Kaiser (2004), dass Technikforscher und Entwickler eine besondere Verantwortung gegenüber der Gesellschaft hinsichtlich der Entwicklung und Einführung neuer Technologien haben. Sie stehen an der Spitze der technischen Entwicklung und haben dadurch mehr Wissen über entstehende Technologien und deren Nutzungsmöglichkeiten, die sich aus ihrem Gebrauch ergeben (Kaiser, 2004). Ethische Aspekte sollten also durchaus bereits bei der Erforschung und Entwicklung von technischen Artefakten bedacht werden, um die Verbreitung solcher Technologien gezielt und vorausschauend begleiten zu können (vgl. Manszeschke et al. 2013). Wo dabei die größten Schwierigkeiten liegen, soll der vorliegende Beitrag beispielhaft aufzeigen.

\section{Methode}

Um die aufgeworfene Fragestellung zu beantworten, wurden die Protokolle von 49 abgeschlossenen Anträgen an die Ethikkommission des Instituts für Psychologie und Arbeitswissenschaft (IPA) der TU Berlin, aus dem Zeitraum von August 2009 bis Mai 2015, hinsichtlich der Häufigkeit und Art der auftretenden Probleme analysiert. Die Anträge stammen alle aus dem Bereich der Erforschung und Entwicklung technischer Systeme. Jeder der Anträge wurde von drei Gutachtern begutachtet. Diese Gutachten waren die Datenbasis für die vorliegende Auswertung. Die von den Gutachtern genannten Probleme wurden in zwei Klassen von Problemen unterteilt und innerhalb der Problemklassen inhaltlich zu Oberkategorien zusammengefasst. Problemklassen waren 1) strukturelle Probleme, wie bspw. eine unzureichende Anonymisierung, bei denen ein Antragsteller bestimmte ethische Aspekte nicht/nicht ausreichend berücksichtigt hatte und 2) Darstellungsprobleme, wie z. B. eine unzureichende Teilnehmerinformation, bei denen der Antragsteller nicht alle Informationen an die Nutzer weitergegeben hatte. Die Oberkategorien unterschieden sich je nach Problemklasse und sind in den Tab. 1 dargestellt. Mehrfachnennungen gleicher Probleme wurden nicht berücksichtigt, allerdings konnte ein Problem auch in beiden Problemklassen auftreten.

\section{Ergebnisse}

Die überblicksartige Betrachtung der Häufigkeitsstatistik zeigt, dass nur 12\% der 49 Anträge ohne problematische Anmerkungen der Gutachter blieben. Bei ca. einem weiteren Drittel der 49 Anträge (36\%) wurden 1-3 Probleme, bei 28\% wurden 4-6 und bei weiteren $22 \%$ wurden 7-9 Probleme von den Gutachtern angemerkt. Hinsichtlich Art der strukturellen Probleme (Tab. 1, linke Spalte) wird deutlich, dass sich die drei häufigsten Herausforderungen auf die Beschreibung des Projekts (65\%), die übermäßige Beanspruchung der potentiellen Nutzer (55\%) oder die nicht ausreichende Anonymisierung von Daten (46\%) bezogen. Ein $\mathrm{Chi}^{2} \mathrm{Test}^{\mathrm{T}}$ auf Gleichverteilung der Problemhäufigkeiten zeigt, dass diese sich nur in der Tendenz über die Oberkategorien dieser Problemklasse verteilen, $\mathrm{Chi}^{2}(5)=10.88, \mathrm{p}<0.10$. 
Vergleichbar zu den strukturellen ethischen Problemen bei der Konzeption von Forschungsund Entwicklungsprojekten wird bei den Darstellungsproblemen deutlich, dass die drei häufigsten Problemfelder die Weitergabe der Informationen an die potentiellen Nutzer (71\%), die ungenaue Beschreibung der Datenverwertung (26\%) und der Umgang mit der Aufwandsentschädigung (20\%) waren (Tab. 1, rechte Spalte). Ein Chi ${ }^{2}$ Test auf Gleichverteilung der Problemhäufigkeiten zeigt, dass diese sich signifikant über die Oberkategorien der Problemklasse verteilen, $\mathrm{Chi}^{2}(5)=58.32, \mathrm{p}<0.01$.

\begin{tabular}{|l|l|l|}
\hline & \multicolumn{2}{|l|}{ Häufigkeiten der Angaben pro Problemklasse } \\
\hline Oberkategorie & 1) Strukturproblem & 2) Darstellungsproblem \\
\hline Projektbeschreibung & $32(65 \%)$ & $35(71 \%)$ \\
\hline Beanspruchung der Nutzer & $27(55 \%)$ & $5(10 \%)$ \\
\hline Anonymisierung von Daten & $23(46 \%)$ & $6(12 \%)$ \\
\hline Datenlöschung/-zugriff & $21(42 \%)$ & $13(26 \%)$ \\
\hline Aufwandsentschädigung & $14(28 \%)$ & $10(20 \%)$ \\
\hline Freiwilligkeit & $6(12 \%)$ & $3(6 \%)$ \\
\hline SUMME & $\mathbf{1 2 3 ( 1 0 0 \% )}$ & $\mathbf{7 2 ( 1 0 0 \% )}$ \\
\hline
\end{tabular}

Tab. 1: Strukturelle- und Darstellungsprobleme bei der Planung von Forschungs- und Entwicklungsvorhaben im Bereich der Mensch-Technik Interaktion

\section{Interpretation \& Diskussion}

Ziel des vorliegenden Beitrages war es, einen empirischen Beitrag zum Ausmaß ethischer Probleme bei der Konzeption von Forschungs- und Entwicklungsprojekten in der MenschMaschine Interaktion zu leisten. Dafür wurden die Gutachten zu 49 Anträgen an die Ethikkommission des IPA der TU Berlin hinsichtlich der geforderten Nachbesserungen der Gutachter ausgewertet. Die Ergebnisse zeigen, dass weniger als 10\% der begutachteten Projekte alle für sie relevanten ethischen Aspekte ausreichend berücksichtigen. Der größte Anteil der strukturellen und Darstellungsprobleme trat bei der Projektbeschreibung auf. So war es vielen Forschern und Entwicklern nicht möglich, ihr Vorhaben so zu beschreiben, dass bspw. die Durchführung oder die verwendeten technischen Geräte und deren Einfluss auf den Nutzer deutlich waren. Eine mögliche Ursache für diese Herausforderung kann in der mangelnden Verbreitung des Wissens über ethisch relevante Aspekte in der MTI Forschung und Entwicklung liegen (vgl. Ropohl 1996). Trotz vieler Informationsmaterialien und Leitfäden zum Thema, scheinen die praktisch relevanten Kerninformationen nicht allen Betroffenen geläufig zu sein. Möglicherweise fehlt es an einer praktisch handhabbaren Gebrauchsethik, die einfach zur Artefaktentwicklung hinzugezogen werden kann (vgl. Manzeschke et al. 2013). Der hier skizzierte Beginn einer empirischen Auseinandersetzung mit dem Thema, könnte dazu dienen, Schwerpunkte einer Gebrauchsethik zu setzen. Ein weiterer inhaltlicher Block von Problemen bezog sich auf den geplanten Umgang der Antragsteller mit den Daten der Nutzer. Entweder war nur eine unzureichende Anonymisierung der Daten gegeben, oder 
die Nutzerdaten sollten (ohne dies zu kommunizieren) an Dritte weitergegeben werden. Damit zeigen die Ergebnisse der Erhebung unter anderem, dass die geforderte Transparenz von Forschungs- und Entwicklungsprojekten in der Mensch-Technik Interaktionsforschung nicht ohne weiteres gegeben ist (vgl. Resnik 2005).

Der vorliegende Beitrag stellt die Problematik natürlich nur ausschnitthaft dar. Trotzdem liefert er einen Beitrag für eine empirische Grundlage der Diskussion über die Bedeutung ethischer Aspekte in der MTI. Weitere Erhebungen sollten die Datenbasis erweitern und nach Gründen für die mangelnde Beachtung ethischer Grundlagen suchen. Darüber hinaus sollte eine praktisch handhabbare Gebrauchsethik entwickeln werden (vgl. Manzeschke et al. 2013), um Forscher und Entwickler überschaubare (ethische) Leitplanken für ihre Arbeit mitzugeben.

\section{Literaturverzeichnis}

de Montjoye, Y.-A., Radaelli, L., Singh, V. K. \& Pentland, A. S. (2015). Unique in the shopping mall: On the reidentifiability of credit card metadata. science (347)6221, 536-539.

Kaiser, M. (2004). Ethics, scienence and precaution: A viewpoint from Norway. Bericht des National Committees for ethics research, (NENT), Oslo: Norway.

Manzeschke, A., Weber, K., Rother, E. \& Fangerau, H. (2013). Ergebnisse der Studie „Ethische Fragen im Bereich Altersgerechter Assistenzsysteme“. Thiel Gruppe: Ludwigsfelde.

Mundt, T., Krüger, F. \& Wollenberg, T. (2012). Who refuses to wash hands? - Privacy issues in modern house installation networks. In Proceedings of the IEEE 2012 Seventh International Conference on Broadband, Wireless Computing, Communication and Applications, 271-277.

Resnik, D. B. (2005). The ethics of science: An introduction. Routledge: New York.

Ropohl, G. (1996). Ethik und Technikbewertung. Suhrkamp: Frankfurt a. Main.

\section{Kontaktinformationen}

Stefan Brandenburg

Kognitionspsychologie und Kognitive Ergonomie

Institut für Psychologie und Arbeitswissenschaft

Sekr. MAR 3-2

Marchstr. 23 (Raum 3.080)

10587 Berlin

Email: stefan.brandenburg@tu-berlin.de 